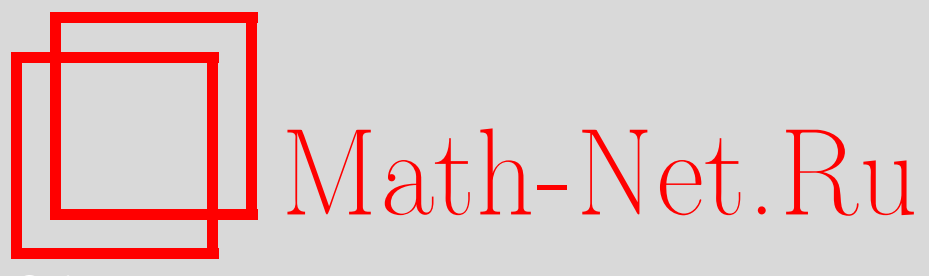

Ю. Х. Эшкабилов, Об одном дискретном "трехчастичном" операторе Шредингера в модели Хаббарда, $T M \Phi$, 2006, том 149, номер 2, 228-243

DOI: https://doi.org/10.4213/tmf4229

Использование Общероссийского математического портала Math-Net.Ru подразумевает, что вы прочитали и согласны с пользовательским соглашением http://www . mathnet.ru/rus/agreement

Параметры загрузки:

IP : 18.209 .158 .208

26 апреля 2023 г., 14:47:05

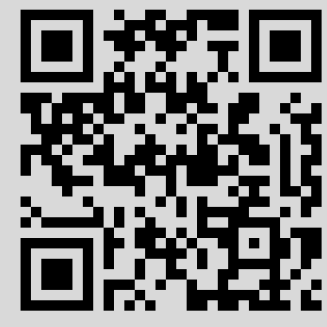




\title{
ОБ ОДНОМ ДИСКРЕТНОМ “ТРЕХЧАСТИЧНОМ” ОПЕРАТОРЕ ШРЕДИНГЕРА В МОДЕЛИ ХАББАРДА
}

\begin{abstract}
В пространстве $L_{2}\left(T^{\nu} \times T^{\nu}\right)$, где $T^{\nu}-\nu$-мерный тор, изучены спектральные свойства "трехчастичного" дискретного оператора Шредингера $\widehat{H}=H_{0}+$ $H_{1}+H_{2}$, где $H_{0}$ - оператор умножения на функцию, $H_{1}, H_{2}$ - частичные интегральные операторы. Доказаны теоремы о существенном спектре оператора $\widehat{H}$. Изучены дискретный и существенный спектры гамильтонианов $H^{\mathrm{t}}$ и $\mathbf{h}$, возникающих в модели Хаббарда на трехмерной решетке.
\end{abstract}

Ключевые слова: дискретный оператор Шредингера, модель Хаббарда, дискретный и существенный спектры дискретных операторов.

\section{1. ВВЕДЕНИЕ}

В настоящее время модель Хаббарда [1]-[3] является одной из наиболее интенсивно изучаемых многоэлектронных моделей металла. Однако до сих пор получено очень мало точных результатов для спектра и волновых функций кристалла, описываемого этой моделью. В настоящее время представляет интерес получение точных результатов хотя бы в частных случаях. В работе [2] была изучена задача о спектре и волновых функциях в простейшем многоэлектронном случае: для двух электронов в трехмерной решетке. Известно, что двухэлектронные системы могут находиться в двух состояниях: триплетном и синглетном [2], [4]. Доказано, что спектр оператора триплета $H_{0}^{\mathrm{t}}$ состоит из единственного отрезка $[2 m, 2 M]$, а у оператора синглета $H^{\mathrm{s}}$ для некоторых значений квазиимпульса существует единственное связанное состояние [2].

В настоящей статье рассматриваются вопросы о возмущении спектра оператора триплета $H^{\mathrm{t}}$ модели, в которой на решетке появляется примесь. Исследуется система, состоящая из двух свободно движущихся электронов и одной примеси на трехмерной решетке, т.е. изучается “трехчастичная" задача: два электрона и одна "примесь" на решетке. Следует отметить некоторые новые черты этой системы, более сложные по сравнению с беспримесным случаем. В беспримесном случае оператор триплета $H_{0}^{\mathrm{t}}$ является унитарно-эквивалентным оператору умножения на функцию [2], и дискретный уровень в спектре гамильтониана $H_{0}^{\mathrm{t}}$ отсутствует. Когда

*Национальный университет Узбекистана, Ташкент, Узбекистан. E-mail: yusup62@rambler.ru 
на решетке имеется примесь, структура гамильтониана значительно усложняется, и оператор триплета $H^{\mathrm{t}}$ приобретает вид $H^{\mathrm{t}}=H_{0}^{\mathrm{t}}+T_{1}+T_{2}$, где $T_{1}, T_{2}$ - частичные интегральные операторы. Поэтому изучение спектра гамильтониана $H^{\mathrm{t}}$ является более интересным с физической и математической точек зрения.

При изучении существенного и дискретного спектров гамильтониана $H^{\mathrm{t}}$ мы сделаем ряд заключений общего характера, касающихся дискретного спектра оператора $H^{\mathrm{t}}$. Поэтому сначала опишем гамильтониан общего вида (который обозначен у нас через $\widehat{H}$ ) и введем некоторые обозначения (раздел 2). В разделе 3 доказаны теоремы о существенном спектре общего “трехчастичного" дискретного оператора Шредингера $\widehat{H}$ (т.е. дано полное описание существенного спектра оператора $\widehat{H}$ ) при общих предположениях для ядер частичных интегральных операторов в $\widehat{H}$. В разделе 4 определены "трехчастичный” и “двухчастичный” дискретные операторы Шредингера $H^{\mathrm{t}}$ и $\mathbf{h}$, возникающие в модели Хаббарда. Доказано, что при некоторых значениях параметров гамильтониана в спектре гамильтониана $H^{\mathrm{t}}$ в дополнение к отрезку $[2 m, 2 M]$ появляются одна ветвь и один дискретный уровень или две ветви и два или три дискретных уровня. Приведено достаточное условие, при котором в спектре гамильтониана $H^{\mathrm{t}}$ отсутствует дискретный уровень.

\section{2. ОПИСАНИЕ ОПЕРАТОРА $\widehat{H}$. НЕКОТОРЫЕ ОБОЗНАЧЕНИЯ}

Пусть $\Omega=T^{\nu}-\nu$-мерный тор в $\mathbb{R}^{\nu}$. Обозначим через $\mu$ меру на $\Omega$ и определим меру $\widehat{\mu}$ на $\Omega \times \Omega$ с помощью равенства $\widehat{\mu}=\mu \otimes \mu$. В пространстве $\mathcal{H}=L_{2}(\Omega \times \Omega)$ рассмотрим ограниченный самосопряженный оператор

$$
\widehat{H}=H_{0}+H_{1}+H_{2},
$$

где действие операторов $H_{0}, H_{1}$ и $H_{2}$ определено равенствами

$$
\begin{aligned}
& H_{0} f(x, y)=h_{0}(x, y) f(x, y), \quad f \in \mathcal{H}, \\
& H_{1} f(x, y)=\int_{\Omega} h_{1}(x, s, y) f(s, y) d s, \quad f \in \mathcal{H}, \\
& H_{2} f(x, y)=\int_{\Omega} h_{2}(x, t, y) f(x, t) d t, \quad f \in \mathcal{H} .
\end{aligned}
$$

Здесь $h_{0}(x, y)$ - вещественнозначная непрерывная функция на $\Omega \times \Omega, h_{1}(x, s, y)$ и $h_{2}(x, t, y)$ - непрерывные функции на $\Omega^{3}$, причем $h_{1}(x, s, y)=\overline{h_{1}(s, x, y)}$ и $h_{2}(x, t, y)=$ $\overline{h_{2}(x, y, t)}$. Операторы $H_{1}$ и $H_{2}$ являются частичными интегральными операторами.

В дальнейшем, если в интегралах не указана область интегрирования, будем понимать это как интегрирование по $\Omega$. Обозначим через $\rho(\cdot), \sigma(\cdot), \sigma_{\mathrm{ess}}(\cdot)$ и $\sigma_{\mathrm{disc}}(\cdot)$ соответственно резольвентное множество, спектр, существенный спектр и дискретный спектр самосопряженных операторов [5].

Для каждого $n \in \mathbb{N}$ введем следующие обозначения:

$$
\begin{aligned}
& K^{(1)}\left(\begin{array}{lllll}
x_{1}, & x_{2}, & \ldots, & x_{n} ; & y \\
s_{1}, & s_{2}, & \ldots, & s_{n} ; & \lambda
\end{array}\right)=
\end{aligned}
$$

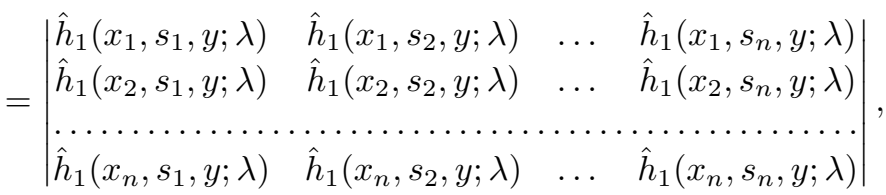


где

$$
\begin{aligned}
& \hat{h}_{1}(x, s, y ; \lambda)=\frac{h_{1}(x, s)}{h_{0}(s, y)-\lambda}, \quad \lambda \in \rho\left(H_{0}\right) ; \\
& K^{(2)}\left(\begin{array}{lllll}
y_{1}, & y_{2}, & \ldots, & y_{n} ; & x \\
t_{1}, & t_{2}, & \ldots, & t_{n} ; & \lambda
\end{array}\right)=
\end{aligned}
$$

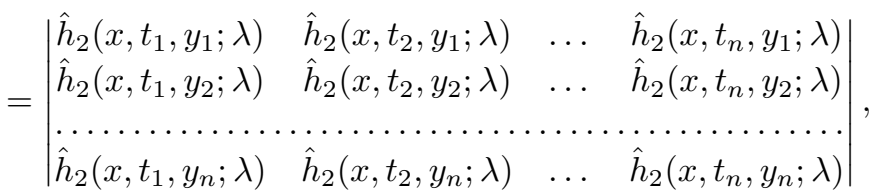

где

$$
\hat{h}_{2}(x, t, y ; \lambda)=\frac{h_{2}(y, t)}{h_{0}(x, t)-\lambda}, \quad \lambda \in \rho\left(H_{0}\right) .
$$

Для каждого $\lambda \in \rho\left(H_{0}\right)$ определим последовательности непрерывных функций $d_{n}^{(1)}(y ; \lambda)$ и $d_{n}^{(2)}(x ; \lambda)$ на $\Omega, n \in \mathbb{N}$, следующим равенством:

$$
d_{n}^{(k)}(\tau ; \lambda)=\int \ldots \int K^{(k)}\left(\begin{array}{ccccc}
s_{1}, & s_{2}, & \ldots, & s_{n} ; & \tau \\
s_{1}, & s_{2}, & \ldots, & s_{n} ; & \lambda
\end{array}\right) d s_{1} d s_{2} \ldots d s_{n}, \quad \tau \in \Omega, \quad k=1,2 .
$$

Также для каждого $\lambda \in \rho\left(H_{0}\right)$ определим последовательности непрерывных функций $M_{n}^{(1)}(x, s, y ; \lambda)$ и $M_{n}^{(2)}(x, s, y ; \lambda)$ на $\Omega^{3}, n \in \mathbb{N}$ :

$$
\begin{aligned}
& M_{n}^{(1)}(x, s, y ; \lambda)=\int \cdots \int K^{(1)}\left(\begin{array}{llllll}
x, & \xi_{1}, & \xi_{2}, & \ldots, & \xi_{n} ; & y \\
s, & \xi_{1}, & \xi_{2}, & \ldots, & \xi_{n} ; & \lambda
\end{array}\right) d \xi_{1} d \xi_{2} \ldots d \xi_{n} \\
& M_{n}^{(2)}(x, s, y ; \lambda)=\int \cdots \int K^{(2)}\left(\begin{array}{llllll}
y, & \xi_{1}, & \xi_{2}, & \ldots, & \xi_{n} ; & x \\
s, & \xi_{1}, & \xi_{2}, & \ldots, & \xi_{n} ; & \lambda
\end{array}\right) d \xi_{1} d \xi_{2} \ldots d \xi_{n} .
\end{aligned}
$$

Очевидно, что при каждом $\lambda \in \rho\left(H_{0}\right)$ функциональные ряды

$$
\sum_{n \in \mathbb{N}} \frac{d_{n}^{(1)}(y ; \lambda)}{n !}, \quad \sum_{n \in \mathbb{N}} \frac{d_{n}^{(2)}(x ; \lambda)}{n !}
$$

абсолютно сходятся на $\Omega$. Следовательно, мы можем определить непрерывные функции $D_{1}(y ; \lambda)$ и $D_{2}(x ; \lambda)$ на $\Omega$ с помощью следующих равенств:

$$
D_{k}(\cdot, \lambda)=1+\sum_{n \in \mathbb{N}} \frac{d_{n}^{(k)}(\cdot ; \lambda)}{n !}, \quad k=1,2 .
$$

Также для каждого $\lambda \in \rho\left(H_{0}\right)$ определим непрерывные функции $M_{1}(x, s, y ; \lambda)$ и $M_{2}(x, s, y ; \lambda)$ на $\Omega^{3}$ такие, что

$$
M_{k}(x, s, y ; \lambda)=\hat{h}_{k}(x, s, y ; \lambda)+\sum_{n \in \mathbb{N}} \frac{M_{n}^{(k)}(x, s, y ; \lambda)}{n !}, \quad k=1,2 .
$$

Введем обозначения

$$
\begin{aligned}
& \sigma_{1}=\left\{\lambda \in \rho\left(H_{0}\right): D_{1}\left(y_{0} ; \lambda\right)=0 \text { для некоторого } y_{0} \in \Omega\right\}, \\
& \sigma_{2}=\left\{\lambda \in \rho\left(H_{0}\right): D_{2}\left(x_{0} ; \lambda\right)=0 \text { для некоторого } x_{0} \in \Omega\right\} .
\end{aligned}
$$


Пусть $R_{0}=R_{0}(\lambda)=\left(H_{0}-\lambda\right)^{-1}, \lambda \in \rho\left(H_{0}\right)$. Для каждого $\lambda \in \rho\left(H_{0}\right) \backslash \sigma_{1}$ резольвента $\left(\widehat{H}_{1}-\lambda\right)^{-1}$ оператора $\widehat{H}_{1}=H_{0}+H_{1}$ имеет вид

$$
\left(\widehat{H}_{1}-\lambda\right)^{-1}=R_{0}\left(E-A_{\lambda}\right)
$$

где $E$ - тождественный оператор в $\mathcal{H}$, оператор $A_{\lambda}$ действует в $\mathcal{H}$ по формуле

$$
A_{\lambda} f(x, y)=\int \frac{M_{1}(x, s, y ; \lambda)}{D_{1}(y ; \lambda)} f(s, y) d s
$$

и $\sigma\left(\widehat{H}_{1}\right)=\sigma\left(H_{0}\right) \cup \sigma_{1}$ (см. работу [6]).

Аналогично при $\lambda \in \sigma\left(H_{0}\right) \backslash \sigma_{2}$ для оператора $\widehat{H}_{2}=H_{0}+H_{2}$ мы имеем

$$
\left(\widehat{H}_{2}-\lambda\right)^{-1}=R_{0}\left(E-B_{\lambda}\right)
$$

где оператор $B_{\lambda}$ в $\mathcal{H}$ действует по формуле

$$
B_{\lambda} f(x, y)=\int \frac{M_{2}(x, s, y ; \lambda)}{D_{2}(x ; \lambda)} f(x, s) d s
$$

и $\sigma\left(\widehat{H}_{2}\right)=\sigma\left(H_{0}\right) \cup \sigma_{2}$.

Пусть $\Pi=\rho\left(H_{0}\right) \cap \rho\left(\widehat{H}_{1}\right) \cap \rho\left(\widehat{H}_{2}\right)$. Для каждого $\lambda \in \Pi$ оператор

$$
K_{\lambda}=\left(H_{1}\left(\widehat{H}_{2}-\lambda\right)^{-1} H_{2}+H_{2}\left(\widehat{H}_{1}-\lambda\right)^{-1} H_{1}\right) R_{0}(\lambda)
$$

является компактным в $\mathcal{H}$. Обозначим $\Delta(\lambda)$ детерминант Фредгольма оператора $E-K_{\lambda}, \lambda \in \Pi$, пусть также $\Pi_{0}=\{\lambda \in \Pi: \Delta(\lambda)=0\}$.

\section{3. СУЩЕСТВЕННЫЙ СПЕКТР ОПЕРАТОРА $\widehat{H}$}

Теорема 3.1. Имеет место следующее включение: $\sigma_{0} \cup \sigma_{1} \cup \sigma_{2} \subset \sigma_{\mathrm{ess}}(\widehat{H})$, где $\sigma_{0}=\sigma\left(H_{0}\right)$.

ДоказАтельство. Сначала докажем, что $\sigma_{0} \subset \sigma_{\mathrm{ess}}(\widehat{H})$. Имеем $\sigma_{0}=[m, M]$, где $m=\inf h_{0}(x, y)$ и $M=\sup h_{0}(x, y)$. Пусть $\lambda_{0} \in[m, M]$. Тогда в силу непрерывности функции $h_{0}(x, y)$ на $\Omega \times \Omega$ существует точка $\left(x_{0}, y_{0}\right) \in \Omega \times \Omega$ такая, что $\lambda_{0}=h_{0}\left(x_{0}, y_{0}\right)$. Пусть

$$
\begin{aligned}
V_{n}\left(x_{0}\right)= & \left\{x \in \Omega: \frac{1}{n+1}<\rho\left(x, x_{0}\right)<\frac{1}{n}\right\}, \quad x_{0} \in \Omega, \\
& W_{n}\left(x_{0}, y_{0}\right)=V_{n}\left(x_{0}\right) \times V_{n}\left(y_{0}\right), \\
& \chi_{W_{n}}(x, y)=\chi_{V_{n}\left(x_{0}\right)}(x) \chi_{V_{n}\left(y_{0}\right)}(y),
\end{aligned}
$$

где $\rho(x, y)$ - метрика в $\mathbb{R}^{\nu}, \chi_{G}(x)$ - характеристическая функция множества $G \subset \mathbb{R}^{\nu}$.

Рассмотрим последовательность ортонормированных функций

$$
f_{n}(x, y)=\frac{\chi_{W_{n}}(x, y)}{\sqrt{\mu\left(V_{n}\left(x_{0}\right)\right) \mu\left(V_{n}\left(y_{0}\right)\right)}} \in \mathcal{H}, \quad n \in \mathbb{N} .
$$


Имеем

$$
\begin{gathered}
\left\|\left(\widehat{H}-\lambda_{0}\right) f_{n}\right\| \leqslant \sup _{x, y \in W_{n}}\left|h_{0}(x, y)-\lambda_{0}\right|+\max _{x, s \in \Omega}\left|h_{1}(x, s, y)\right| \sqrt{\mu(\Omega) \mu\left(V_{n}\left(x_{0}\right)\right)}+ \\
\quad+\max _{y, t \in \Omega}\left|h_{2}(x, s, y)\right| \sqrt{\mu(\Omega) \mu\left(V_{n}\left(y_{0}\right)\right)} \rightarrow 0
\end{gathered}
$$

при $n \rightarrow \infty$.

Действительно, при $n \rightarrow \infty$ имеем сходимость $(x, y) \rightarrow\left(x_{0}, y_{0}\right)$, из непрерывности функции $h_{0}(x, y)$ на $\Omega \times \Omega$ вытекает, что $h_{0}(x, y) \rightarrow h_{0}\left(x_{0}, y_{0}\right)=\lambda_{0}$, а остальные слагаемые стремятся к нулю в силу того, что $\mu\left(V_{n}\left(x_{0}\right)\right) \rightarrow 0$ и $\mu\left(V_{n}\left(y_{0}\right)\right) \rightarrow 0$ при $n \rightarrow \infty$. Отсюда и из критерия Вейля о существенном спектре [5] получим $\sigma_{0} \subset$ $\sigma_{\text {ess }}(\widehat{H})$.

Теперь докажем, что $\sigma_{1} \subset \sigma_{\text {ess }}(\widehat{H})$. Пусть $\lambda_{0} \in \sigma_{1}$. Тогда существует элемент $\alpha_{0} \in \Omega$ такой, что $D_{1}\left(\alpha_{0} ; \lambda_{0}\right)=0$. При каждом $\alpha \in \Omega$ обозначим через $Q_{\alpha}=Q_{\alpha}(\lambda)$, $\lambda \in \rho\left(H_{0}\right)$, компактный оператор в $\mathcal{H}_{0}=L_{2}(\Omega)$ с непрерывным ядром

$$
k_{\alpha}(x, s ; \lambda)=\hat{h}_{1}(x, s, \alpha ; \lambda)=\frac{h_{1}(x, s, \alpha)}{h_{0}(s, \alpha)-\lambda} .
$$

Тогда для детерминанта $\Delta_{\alpha}^{(1)}(\lambda)$ оператора $I+Q_{\alpha}(\lambda), \lambda \in \rho\left(H_{0}\right)$, где $I$ - тождественный оператор в $\mathcal{H}_{0}$, имеем $\Delta_{\alpha}^{(1)}(\lambda)=D_{1}(\alpha ; \lambda)[7]$. Отсюда получим, что $\Delta_{\alpha_{0}}^{(1)}\left(\lambda_{0}\right)=0$, т.е. число -1 является собственным значением оператора $Q_{\alpha_{0}}\left(\lambda_{0}\right)$. Следовательно, существует вектор $\varphi \in \mathcal{H}_{0},\|\varphi\|=1$, такой, что $Q_{\alpha_{0}}\left(\lambda_{0}\right) \varphi(x)=-\varphi(x)$.

Пусть $\chi_{n}(y)$ - характеристическая функция множества $V_{n}=V_{n}\left(\alpha_{0}\right)$. Определим последовательность ортонормированных функций

$$
f_{n}(x, y)=\frac{\gamma_{n}}{\sqrt{\mu\left(V_{n}\right)}} \frac{\varphi(x) \chi_{n}(y)}{h_{0}(x, y)-\lambda_{0}} \in \mathcal{H}, \quad n \in \mathbb{N},
$$

где $\left\{\gamma_{n}\right\}$ - некоторая ограниченная числовая последовательность, которая обеспечивает нормированность последовательности $\left\{f_{n}\right\}$.

Имеем

$$
\left(\widehat{H}-\lambda_{0}\right) f_{n}(x, y)=\frac{\gamma_{n} \chi_{n}(y)}{\sqrt{\mu\left(V_{n}\right)}}\left(\varphi(x)+Q_{y}\left(\lambda_{0}\right) \varphi(x)\right)+H_{2} f_{n}(x, y) .
$$

Отметим, что при фиксированном $\lambda \in \rho\left(H_{0}\right)$

$$
\lim _{\alpha \rightarrow \alpha_{0}}\left\|Q_{\alpha}(\lambda)-Q_{\alpha_{0}}(\lambda)\right\|_{\mathcal{H}_{0}}=0
$$

и существует число $a>0$ такое, что $\min _{x, t \in \Omega}\left|h_{0}(x, t)-\lambda_{0}\right| \geqslant a>0$ и

$$
\left\|H_{2} f_{n}\right\| \leqslant \frac{\gamma_{n}}{a} \max _{x, y, t \in \Omega}\left|h_{2}(x, t, y)\right| \sqrt{\mu(\Omega) \mu\left(V_{n}\right)}, \quad n \in \mathbb{N} .
$$

Следовательно,

$$
\left\|\left(\widehat{H}-\lambda_{0}\right) f_{n}\right\| \leqslant \gamma_{n}\left(\sqrt{\sup _{\alpha \in \overline{V_{n}\left(x_{0}\right)}}\left\|\varphi+Q_{\alpha}\left(\lambda_{0}\right) \varphi\right\|_{\mathcal{H}_{0}}^{2}}+C \sqrt{\mu\left(V_{n}\right)}\right), \quad n \in \mathbb{N},
$$


где $C>0$.

Однако $\lim _{n \rightarrow \infty} \overline{V_{n}\left(\alpha_{0}\right)}=\left\{\alpha_{0}\right\}$. Тогда

$$
\lim _{n \rightarrow \infty} \sup _{\alpha \in \overline{V_{n}\left(\alpha_{0}\right)}}\left\|Q_{\alpha}\left(\lambda_{0}\right) \varphi+\varphi\right\|_{\mathcal{H}_{0}}=\left\|Q_{\alpha_{0}}\left(\lambda_{0}\right) \varphi+\varphi\right\|_{\mathcal{H}_{0}}=0
$$

так как $\varphi$ - собственный вектор оператора $Q_{\alpha_{0}}\left(\lambda_{0}\right)$, соответствующий собственному значению -1. Таким образом, получим, что

$$
\lim _{n \rightarrow \infty}\left\|\left(\widehat{H}-\lambda_{0}\right) f_{n}\right\|=0,
$$

т.е. $\lambda_{0} \in \sigma_{\text {ess }}(\widehat{H})$. Следовательно, $\sigma_{1} \subset \sigma_{\mathrm{ess}}(\widehat{H})$. Аналогично доказывается, что $\sigma_{2} \subset \sigma_{\text {ess }}(\widehat{H})$. Доказательство теоремы 3.1 завершено.

Лемма 3.1. Если $\lambda \in \Pi \backslash \Pi_{0}$, то оператор

$$
N_{\lambda}=-\left(H_{0}-\lambda\right)^{-1}+\left(\widehat{H}_{1}-\lambda\right)^{-1}+\left(\widehat{H}_{2}-\lambda\right)^{-1}
$$

действует в $\mathcal{H}$ и ингективен.

ДокАЗАтЕЛЬство. Пусть $\lambda \in \Pi \backslash \Pi_{0}$ и существует ненулевой элемент $f_{0} \in \mathcal{H}$ такой, что $N_{\lambda} f_{0}=\theta$. Положим $\tilde{f}_{0}=R_{0} f_{0}$. Тогда $\tilde{f}_{0} \in \mathcal{H}$ и $\tilde{f}_{0} \neq \theta$, поскольку $\lambda \notin \sigma\left(H_{0}\right)$. Для оператора $N_{\lambda}$ имеет место равенство

$$
N_{\lambda}=\left(E-\left(\widehat{H}_{1}-\lambda\right)^{-1} H_{1}-\left(\widehat{H}_{2}-\lambda\right)^{-1} H_{2}\right) R_{0}(\lambda), \quad \lambda \in \Pi .
$$

Из $N_{\lambda} f_{0}=\theta$ следует, что $\tilde{f}_{0}-\left(\widehat{H}_{1}-\lambda\right)^{-1} H_{1} \tilde{f}_{0}=\left(\widehat{H}_{2}-\lambda\right)^{-1} H_{2} \tilde{f}_{0}$, т.е.

$$
\left(\widehat{H}_{1}-\lambda\right)^{-1}\left(\widehat{H}_{1}-\lambda\right) \tilde{f}_{0}-\left(\widehat{H}_{1}-\lambda\right)^{-1} H_{1} \tilde{f}_{0}=\left(\widehat{H}_{2}-\lambda\right)^{-1} H_{2} \tilde{f}_{0} .
$$

Значит, $\left(\widehat{H}_{1}-\lambda\right)^{-1} f_{0}=\left(\widehat{H}_{2}-\lambda\right)^{-1} H_{2} R_{0} f_{0}$. Аналогично получим, что $\left(\widehat{H}_{2}-\lambda\right)^{-1} f_{0}=$ $\left(\widehat{H}_{1}-\lambda\right)^{-1} H_{1} R_{0} f_{0}$. Следовательно,

$$
\begin{aligned}
K_{\lambda} f_{0} & =H_{1}\left(\widehat{H}_{2}-\lambda\right)^{-1} H_{2} R_{0} f_{0}+H_{2}\left(\widehat{H}_{1}-\lambda\right)^{-1} H_{1} R_{0} f_{0}= \\
& =H_{1}\left(\widehat{H}_{1}-\lambda\right)^{-1} f_{0}+H_{2}\left(\widehat{H}_{2}-\lambda\right)^{-1} f_{0}= \\
& =2 f_{0}-\left(H_{0}-\lambda\right)\left(N_{\lambda}+\left(H_{0}-\lambda\right)^{-1}\right) f_{0}=f_{0},
\end{aligned}
$$

поэтому число 1 является собственным значением оператора $K_{\lambda}$, т.е. $\lambda \in \Pi_{0}$. Это противоречит предположению $\lambda \in \Pi \backslash \Pi_{0}$.

Пусть $L(\mathcal{H})$ - пространство линейных ограниченных операторов в $\mathcal{H}=L_{2}(\Omega \times \Omega)$.

ЛЕмма 3.2. Справедливы следующие утверждения:

а) для каждого $\lambda \in \Pi \backslash \Pi_{0}$ система операторных уравнений

$$
\begin{aligned}
R_{1}\left(\widehat{H}_{1}-\lambda\right) & =-R_{0} H_{1}, \\
R_{2}\left(\widehat{H}_{2}-\lambda\right) & =-R_{0} H_{2}, \\
R(\widehat{H}-\lambda) & =-\left(R_{1} H_{2}+R_{2} H_{1}\right)
\end{aligned}
$$


разрешима в $L(\mathcal{H})$ и оператор $\widetilde{R}=R_{0}+R_{1}+R_{2}+R$ является левым обратным $\kappa$ оператору $\widehat{H}-\lambda$, где $R_{1}=R_{1}(\lambda), R_{2}=R_{2}(\lambda)$ и $R=R(\lambda)$ - решение системы (3.1.1)(3.1.3) ;

б) для каждого $\lambda \in \Pi \backslash \Pi_{0}$ система операторных уравнений

$$
\begin{aligned}
\left(\widehat{H}_{1}-\lambda\right) T_{1} & =-H_{1} R_{0}, \\
\left(\widehat{H}_{2}-\lambda\right) T_{2} & =-H_{2} R_{0}, \\
(\widehat{H}-\lambda) T & =-\left(H_{1} T_{2}+H_{2} T_{1}\right)
\end{aligned}
$$

разрешима в $L(\mathcal{H})$ и оператор $\widetilde{T}=T_{0}+T_{1}+T_{2}+T$ является правым обратным $\kappa$ onepamopy $\widehat{H}-\lambda$, где $T_{0}=R_{0}(\lambda)$ u $T_{1}=T_{1}(\lambda), T_{2}=T_{2}(\lambda), T=T(\lambda)$ - решение системы $\left(3.1 .1^{\prime}\right)-\left(3.1 .3^{\prime}\right)$

ДоказатеЛЬСтво. Пусть $\lambda \in \Pi$. Тогда $\lambda \in \rho\left(\widehat{H}_{1}\right) \cap \rho\left(\widehat{H}_{2}\right)$, поэтому для операторов $\widehat{H}_{1}$ и $\widehat{H}_{2}$ существуют соответственно резольвенты $\left(\widehat{H}_{1}-\lambda\right)^{-1} \in L(\mathcal{H})$ и $\left(\widehat{H}_{2}-\lambda\right)^{-1} \in L(\mathcal{H})$. Из уравнений (3.1.1) и (3.1.2) находим, что при $\lambda \in \Pi$

$$
\begin{aligned}
& R_{1}=R_{1}(\lambda)=-R_{0} H_{1}\left(\widehat{H}_{1}-\lambda\right)^{-1} \in L(\mathcal{H}), \\
& R_{2}=R_{2}(\lambda)=-R_{0} H_{2}\left(\widehat{H}_{2}-\lambda\right)^{-1} \in L(\mathcal{H}) .
\end{aligned}
$$

При $\lambda_{0} \in \Pi \backslash \Pi_{0}$, умножив обе части уравнения (3.1.3) справа на инъективный оператор

$$
\widehat{N}_{1}(\lambda)=\left(E-\left(\widehat{H}_{1}-\lambda\right)^{-1} H_{1}-\left(\widehat{H}_{2}-\lambda\right)^{-1} H_{2}\right) R_{0}(\lambda)=N_{\lambda},
$$

получим

$$
\begin{aligned}
R(\widehat{H}-\lambda) \widehat{N}_{1}=R & +R\left(\left(\widehat{H}_{1}-\lambda\right) \widetilde{R}_{1}+H_{1} R_{0}\right)+R\left(\left(\widehat{H}_{2}-\lambda\right) \widetilde{R}_{2}+H_{2} R_{0}\right)+ \\
& +R\left(H_{1} \widetilde{R}_{2}+H_{2} \widetilde{R}_{1}\right)=-\left(R_{1} H_{2}+R_{2} H_{1}\right) \widehat{N}_{1}(\lambda),
\end{aligned}
$$

где $\widetilde{R}_{k}=\widetilde{R}_{k}(\lambda)=-\left(\widehat{H}_{k}-\lambda\right)^{-1} H_{k} R_{0}(\lambda), k=1,2$.

Отсюда получим уравнение

$$
R\left(E+H_{1} \widetilde{R}_{2}+H_{2} \widetilde{R}_{1}\right)=-\left(R_{1} H_{2}+R_{2} H_{1}\right) \widehat{N}_{1},
$$

т.е. $R\left(E-K_{\lambda}\right)=P_{\lambda} N_{\lambda}$, где

$$
P_{\lambda}=-\left(R_{1}(\lambda) H_{2}+R_{2}(\lambda) H_{1}\right) .
$$

В силу обратимости оператора $E-K_{\lambda}$ имеем

$$
R=R(\lambda)=P_{\lambda} N_{\lambda}\left(E-K_{\lambda}\right)^{-1} \in L(\mathcal{H}) .
$$

Итак, мы доказали разрешимость системы операторных уравнений (3.1.1)-(3.1.3) при $\lambda \in \Pi \backslash \Pi_{0}$. Легко проверить, что для оператора $\widetilde{R}=R_{0}+T_{1}+R_{2}+R$ выполняется равенство $\widetilde{R}(\widehat{H}-\lambda)=E$ при всех $\lambda \in \Pi \backslash \Pi_{0}$. Первое утверждение леммы доказано.

Аналогично доказывается второе утверждение, однако решение системы уравнений $\left(3.1 .1^{\prime}\right)-\left(3.1 .3^{\prime}\right)$ имеет вид

$$
\begin{aligned}
T_{1} & =T_{1}(\lambda)=-\left(\widehat{H}_{1}-\lambda\right)^{-1} H_{1} R_{0}(\lambda), & & \lambda \in \rho\left(H_{0}\right) \cap \rho\left(H_{1}\right), \\
T_{2} & =T_{2}(\lambda)=-\left(\widehat{H}_{2}-\lambda\right)^{-1} H_{2} R_{0}(\lambda), & & \lambda \in \rho\left(H_{0}\right) \cap \rho\left(H_{2}\right), \\
T & =T(\lambda)=\left(E-P_{\lambda}\right)^{-1} N_{\lambda} K_{\lambda}, & & \lambda \in \Pi \backslash \Pi_{0} .
\end{aligned}
$$


Отметим, что оператор $P_{\lambda}, \lambda \in \Pi$, заданный формулой (3.2), является компактным в $\mathcal{H}$, и имеет место равенство $P_{\lambda}=R_{0} K_{\lambda} R_{0}^{-1}, \lambda \in \Pi$.

В силу леммы 3.2 для каждого $\lambda \in \Pi \backslash \Pi_{0}$ существуют левый и правый обратные операторы к $\widehat{H}-\lambda$. Следовательно, для резольвенты $R_{\lambda}(\widehat{H}), \lambda \in \Pi \backslash \Pi_{0}$, оператора $\widehat{H}$ имеем

$$
R_{\lambda}(\widehat{H})=R_{0}(\lambda)+R_{1}(\lambda)+R_{2}(\lambda)+R(\lambda)=T_{0}(\lambda)+T_{1}(\lambda)+T_{2}(\lambda)+T(\lambda) .
$$

Отсюда следует

ТЕОрема 3.2. При любом $\lambda \in \Pi \backslash \Pi_{0}$ для резольвенты $R_{\lambda}(\widehat{H})$ оператора $\widehat{H}$ справедлива формула

$$
R_{\lambda}(\widehat{H})=R_{0}+R_{1}+R_{2}+R
$$

¿əe

$$
\begin{aligned}
R_{1} & =R_{1}(\lambda)=-R_{0} H_{1}\left(\widehat{H}_{1}-\lambda\right)^{-1}, \\
R_{2} & =R_{2}(\lambda)=-R_{0} H_{2}\left(\widehat{H}_{2}-\lambda\right)^{-1}, \\
R & =R(\lambda)=P_{\lambda} N_{\lambda}\left(E-K_{\lambda}\right)^{-1} .
\end{aligned}
$$

Лемма 3.3. Если число $\lambda \in \mathbb{C} \backslash\left(\sigma_{0} \cup \sigma_{1} \cup \sigma_{2}\right)$ - собственное значение оператора $\widehat{H}$, то число 1 является собственным значением компактного оператора $K_{\lambda}$.

ДокАЗАтеЛьство. Легко заметить, что $\Pi=\mathbb{C} \backslash\left(\sigma_{0} \cup \sigma_{1} \cup \sigma_{2}\right)$. Пусть $\lambda \in \Pi-$ собственное значение оператора $\widehat{H}$ и $f_{0} \in \mathcal{H}, f_{0} \neq \theta,-$ соответствующий собственный вектор, т.е. $(\widehat{H}-\lambda) f_{0}=\theta$. При этом

$$
\left(R_{0}+R_{1}+R_{2}\right)(\widehat{H}-\lambda)=E+R_{1} H_{2}+R_{2} H_{1}=E-P_{\lambda}, \quad \lambda \in \Pi .
$$

Следовательно, $\left(E-P_{\lambda}\right) f_{0}=\theta$, т.е. число 1 - собственное значение оператора $P_{\lambda}$. Тогда число 1 является также собственным значением оператора $K_{\lambda}$. Лемма 3.3 доказана.

Теорема 3.3. Имеет место равенство

$$
\sigma_{\text {ess }}(\widehat{H})=\sigma\left(H_{0}\right) \cup \sigma\left(\widehat{H}_{1}\right) \cup\left(\widehat{H}_{2}\right)=\sigma\left(\widehat{H}_{1}\right) \cup \sigma\left(\widehat{H}_{2}\right)=\sigma_{0} \cup \sigma_{1} \cup \sigma_{2} .
$$

ДоказАтЕЛЬСтво. Из теоремы 3.1 мы имеем $\sigma_{0} \cup \sigma_{1} \cup \sigma_{2} \subset \sigma_{\text {ess }}(\widehat{H})$, и достаточно показать обратное включение. В силу теоремы 3.2 для каждого $\lambda \in \Pi \backslash \Pi_{0}$ существует резольвента $R_{\lambda}(\widehat{H}) \in L(\mathcal{H})$. Следовательно, $\sigma(\widehat{H}) \subset \mathbb{C} \backslash\left(\Pi \backslash \Pi_{0}\right)=\sigma_{0} \cup \sigma_{1} \cup \sigma_{2} \cup \Pi_{0}$. Однако произвольная точка $\lambda \in \Pi_{0}$ является изолированной в $\Pi_{0}$, следовательно, она изолирована также в $\sigma_{0} \cup \sigma_{1} \cup \sigma_{2} \cup \Pi_{0}$, и в силу леммы 3.3 число $\lambda$ является конечнократным собственным значением оператора $\widehat{H}$, т.е. $\lambda \in \sigma_{\operatorname{disc}}(\widehat{H})$. Отсюда получим, что $\sigma_{\mathrm{ess}}(\widehat{H}) \subset \sigma_{0} \cup \sigma_{1} \cup \sigma_{2}$.

\section{4. "ТРЕХЧАСТИЧНЫЙ" ОПЕРАТОР ТРИПЛЕТА}

Пусть $\mathbb{Z}^{\nu}-\nu$-мерная целочисленная решетка, $\sigma$ - множество значений спина электрона, т.е. $\sigma=\{-1 / 2,1 / 2\}$. Через $\tilde{l}_{2}=l_{2}\left(\mathbb{Z}^{\nu} \times \sigma\right)$ обозначим гильбертово пространство квадратично-суммируемых функций, определенных на $\mathbb{Z}^{\nu} \times \sigma$, т.е. для любой 
$f \in \tilde{l}$ мы имеем $f=f(m, \gamma)$, где $m \in \mathbb{Z}^{\nu}, \gamma \in \sigma$, и

$$
\sum_{m \in \mathbb{Z}^{\nu}, \gamma \in \sigma}|f(m, \gamma)|^{2}<\infty
$$

Скалярное произведение для функций $f, g \in \tilde{l}_{2}$ определяется по формуле

$$
(f, g)=\sum_{s \in \mathbb{Z}^{\nu} \times \sigma} f(s) \overline{g(s)}, \quad s=(m, \gamma) .
$$

Обозначим через $F$ и $F_{\text {as }}$ соответственно пространство Фока над $\tilde{l}_{2}$ и антисимметричное пространство Фока над $\tilde{l}_{2}[5]$.

Рассмотрим на решетке $\mathbb{Z}^{3}$ два электрона и одну примесь. Допустим, что примесь находится в узле $0 \in \mathbb{Z}^{3}$. Тогда гамильтониан в модели Хаббарда имеет вид [2], [3]

$$
H=\widetilde{H}+H_{0},
$$

где

$$
\begin{aligned}
\widetilde{H} & =\alpha \sum_{\substack{m \in \mathbb{Z}^{3} \\
\gamma=\uparrow, \downarrow}} a_{m \gamma}^{+} a_{m \gamma}+\beta \sum_{\substack{m \in \mathbb{Z}^{3}, \tau= \pm e_{j} \\
\gamma=\uparrow, \downarrow}} a_{m \gamma}^{+} a_{m+\tau, \gamma}+\mu \sum_{m \in \mathbb{Z}^{3}} n_{m \uparrow} n_{m \downarrow}, \\
H_{0} & =\left(\alpha_{0}-\alpha\right) \sum_{\gamma} a_{0 \gamma}^{+} a_{0 \gamma}+\left(\beta_{0}-\beta\right) \sum_{\tau, \gamma}\left(a_{0 \gamma}^{+} a_{\tau \gamma}+a_{\tau \gamma}^{+} a_{0 \gamma}\right)+\left(\mu_{0}-\mu\right) n_{0 \uparrow} n_{0 \downarrow} .
\end{aligned}
$$

Здесь $\alpha_{m \gamma}^{+}\left(a_{m \gamma}\right)$ - оператор рождения (уничтожения) в узле $m \in \mathbb{Z}^{3}$ электрона с проекцией спина $\uparrow, \downarrow$ (через $\uparrow$ и $\downarrow$ обозначены соответственно значения спина $-1 / 2$ и $1 / 2) ; n_{m \gamma}=\alpha_{m \gamma}^{+} \alpha_{m \gamma} ; \pm e_{j}$ - единичные орты, $j=1,2,3 ; \alpha_{0}(\alpha)$ - энергия электрона на примесном (регулярном) узле; $\beta_{0}(\beta)$ - интеграл переноса электрона с примесного (регулярного) узла на ближайший; $\mu_{0}(\mu)$ - параметр кулоновского взаимодействия двух электронов на примесном (регулярном) узле. В дальнейшем для удобства будем считать, что $\beta>0$. Гамильтониан $H$ действует в пространстве $F_{\text {as. }}$.

Пусть $\varphi_{0}$ - вакуумный вектор в пространстве $F$. Триплетному состоянию соответствуют свободное движение двух электронов на решетке и следующие базисные функции [2], [4]:

$$
t_{m n}^{-1}=a_{m \downarrow}^{+} a_{n \downarrow}^{+} \varphi_{0}, \quad t_{m n}^{0}=\frac{1}{\sqrt{2}}\left(a_{m \uparrow}^{+} a_{n \downarrow}^{+}+a_{m \downarrow}^{+} a_{n \uparrow}^{+}\right) \varphi_{0}, \quad t_{m n}^{1}=a_{m \uparrow}^{+} a_{n \uparrow}^{+} \varphi_{0} .
$$

Подпространство $\widetilde{F}_{k}^{\mathrm{t}}, k \in\{-1 ; 0 ; 1\}$, соответствующее триплетному состоянию, состоит из множества всех векторов $\psi$ вида

$$
\psi=\sum_{m, n \in \mathbb{Z}^{3}} \tilde{f}(m, n) t_{m n}^{k}, \quad \tilde{f} \in l_{2}^{\mathrm{as}},
$$

где $l_{2}^{\text {as }}$ - пространство антисимметричных функций из $l_{2}\left(\mathbb{Z}^{3} \times \mathbb{Z}^{3}\right)[2]$.

Одночастичное пространство $\widetilde{F}_{1}$ состоит из всех векторов $\phi$ вида

$$
\phi=\sum_{m \in \mathbb{Z}^{3}} \tilde{f}(m) t_{m}, \quad \tilde{f} \in l_{2}\left(\mathbb{Z}^{3}\right),
$$


где $t_{m}=a_{m \downarrow}^{+} \varphi_{0}, m \in \mathbb{Z}^{3}$.

Рассмотрим ограниченный самосопряженный оператор $H^{\mathrm{t}}$, действующий в $l_{2}^{\text {as }}$ по формуле

$$
\begin{aligned}
\left(H^{\mathrm{t}} f\right)(m, n)=2 \alpha & f(m, n)+\beta \sum_{\tau}(f(m+\tau, n)+f(m, n+\tau))+ \\
& +\varepsilon_{1}\left(\delta_{m 0}+\delta_{n 0}\right) f(m, n)+\varepsilon_{2} \sum_{\tau}\left(\delta_{m 0} f(m+\tau, n)+\delta_{n 0} f(m, n+\tau)\right)+ \\
& +\varepsilon_{2} \sum_{\tau}\left(\delta_{m \tau} f(m-\tau, n)+\delta_{n \tau} f(m, n-\tau)\right)
\end{aligned}
$$

где $\delta_{m n}$ - символ Кронекера, $\tau$ означает суммирование по ближайшим соседям в решетке $\mathbb{Z}^{3}$, т.е. $\tau \in\left\{ \pm e_{j}\right\}, \varepsilon_{1}=\alpha_{0}-\alpha, \varepsilon_{2}=\beta_{0}-\beta$.

Наряду с оператором $H^{\mathrm{t}}$ будем рассматривать самосопряженный оператор $\mathbf{h}$, действующий в пространстве $l_{2}\left(\mathbb{Z}^{3}\right)$ по формуле

$$
\begin{aligned}
(\mathbf{h} f)(m)=\alpha & f(m)+\beta \sum_{\tau} f(m+\tau)+\varepsilon_{1} \delta_{m 0} f(m)+ \\
& +\varepsilon_{2} \sum_{\tau}\left(\delta_{m 0} f(m+\tau)+\delta_{n 0} f(m-\tau)\right) .
\end{aligned}
$$

Лемма 4.1. Пространства $\widetilde{F}_{k}^{\mathrm{t}}, k \in\{-1,0,1\}$, инвариантны относителъно оператора $H$. Операторы $\widetilde{H}_{k}^{\mathrm{t}}=\left.H\right|_{\widetilde{F}_{k}^{\mathrm{t}}}$ являются ограниченными самосопряжсенными $в$ $\widetilde{F}_{k}^{\mathrm{t}}$ и действуют по формуле

$$
\widetilde{H}_{k}^{\mathrm{t}} \psi=\sum_{m, n}\left(H^{\mathrm{t}} f\right)(m, n) t_{m n}^{k}, \quad \psi \in \widetilde{F}_{k}^{\mathrm{t}}
$$

где оператор $H^{\mathrm{t}}$ действует в $l_{2}^{\text {as }}$ в coomветствии $c(4.1)$.

Лемма 4.2. Пространство $\widetilde{F}_{1}$ инвариантно относительно оператора $H$. Oneратор $\widetilde{H}_{1}=\left.H\right|_{\widetilde{F}_{1}}$ является ограниченным самосопряженным в $\widetilde{F}_{k}^{\mathrm{t}}$ и действует по формуле

$$
\widetilde{H}_{1} \phi=\sum_{m}(\mathbf{h} f)(m) t_{m}, \quad \phi \in \widetilde{F}_{1},
$$

где оператор $\mathbf{h}$ определен в (4.2).

Доказательство обеих лемм можно получить, действуя гамильтонианом $H$ на векторы $\psi \in \widetilde{F}_{k}^{\mathrm{t}}$ и $\phi \in \widetilde{F}_{1}$, пользуясь антикоммутационными соотношениями операторов рождения и уничтожения

$$
\left\{a_{m \gamma}, a_{n \beta}^{+}\right\}=\delta_{m n} \delta_{n \beta}, \quad\left\{a_{m \gamma}, a_{n \beta}\right\}=a_{m \gamma}^{+} a_{n \beta}^{+}=\theta,
$$

и свойством $a_{m \gamma}=\theta$.

Из критерия Вейля [5] следует, что

$$
\sigma\left(\widetilde{H}_{k}^{\mathrm{t}}\right)=\sigma\left(H^{\mathrm{t}}\right), \quad k \in\{-1,0,1\}, \quad \sigma\left(\widetilde{H}_{1}\right)=\sigma(\mathbf{h}) .
$$

Таким образом, для анализа спектров операторов $\widetilde{H}_{k}^{\mathrm{t}}$ и $\widetilde{H}_{1}$ достаточно изучить соответственно спектры операторов $H^{\mathrm{t}}(4.1)$ и $\mathbf{h}(4.2)$. 
Оператор $H^{\mathrm{t}}$ будем называть “трехчастичным” оператором триплета (два электрона и одна примесь), а оператор $\mathbf{h}$ - “двухчастичным” оператором (один электрон и одна примесь).

В импульсном представлении операторы $H^{\mathrm{t}}$ и $\mathbf{h}$ действуют соответственно в гильбертовых пространствах $L_{2}^{\text {as }}$ и $L_{2}\left(T^{3}\right)$ по следующим формулам:

$$
\begin{aligned}
H^{\mathrm{t} f} f(x, y)=\{u(x)+u(y)\} f(x, y)+ & \\
& \quad+\int_{T^{3}} \int_{T^{3}}(K(x, s) \delta(y-t)+K(y, t) \delta(x-s)) f(s, t) d s d t, \quad f \in L_{2}^{\mathrm{as}}, \\
\mathbf{h} f(x)= & u(x) f(x)+\int_{T^{3}} K(x, s) f(s) d s, \quad f \in L_{2}\left(T^{3}\right),
\end{aligned}
$$

где $T^{3}$ - трехмерный тор, $L_{2}^{\text {as }}$ - подпространство антисимметричных функций в $L_{2}\left(T^{3} \times T^{3}\right), u(x)=\alpha+\beta h_{0}(x), K(x, s)=\varepsilon_{1}+\varepsilon_{2}\left(h_{0}(x)+h_{0}(s)\right), h_{0}(x)=2 \sum_{j=1}^{3} \cos x_{j}$, $\delta(x)$ - трехмерная дельта-функция Дирака на $T^{3}$.

Пусть $p(x)$ - вещественнозначная непрерывная функция на $T^{\nu}, k(x, s) \in L_{2}\left(T^{\nu} \times\right.$ $\left.T^{\nu}\right)$ и $k(x, s)=\overline{k(s, x)}, x, s \in T^{\nu}$. Рассмотрим операторы $U_{0}$ и $U$ в $L_{2}\left(T^{\nu}\right)$, действующие по формулам

$$
U_{0} f(x)=p(x) f(x), \quad U f(x)=\int_{T^{\nu}} k(x, s) f(s) d s .
$$

В соответствии с теоремой Вейля о существенном спектре [8] существенный спектр самосопряженного оператора $U_{0}+U$ не зависит от ядра $k(x, s)$ и совпадает с отрезком $\left[m_{\nu}, M_{\nu}\right]$, т.е. $\sigma_{\mathrm{ess}}\left(U_{0}\right)=\left[m_{\nu}, M_{\nu}\right]$, где $m_{\nu}=\min _{x \in T^{\nu}} p(x)$ и $M_{\nu}=\max _{x \in T^{\nu}} p(x)$.

ПрЕДЛОЖЕНИЕ 4.1. Число $z \in \mathbb{C} \backslash\left[m_{\nu}, M_{\nu}\right]$ является собственным значением оператора $U_{0}+U$ тогда и только тогда, когда число $\lambda=-1$ является собственным значением оператора $V(z)$, действующего в $L_{2}\left(T^{\nu}\right)$ по формуле

$$
V(z) f(x)=\int_{T^{\nu}} \frac{k(x, y)}{u(y)-z} f(y) d y .
$$

ДоказАтеЛьство. Необходимость. Пусть $z \in \mathbb{C} \backslash\left[m_{\nu}, M_{\nu}\right]$ - собственное значение оператора $U_{0}+U$ и $f \in L_{2}\left(T^{\nu}\right)$ - соответствующая собственная функция, т.е.

$$
u(x) f(x)+\int_{T^{\nu}} k(x, y) f(y) d y=z f(x)
$$

или

$$
(u(x)-z) f(x)+\int_{T^{\nu}} k(x, y) f(y) d y=0 .
$$

Обозначая через $\varphi(x)$ функцию $(u(x)-z) f(x)$, имеем

$$
V(z) \varphi(x)=\int_{T^{\nu}} \frac{k(x, y)}{u(y)-z} \varphi(y) d y=-\varphi(x),
$$

т.е. $\lambda=-1$ есть собственное значение, а $\varphi \in L_{2}\left(T^{\nu}\right)$ - соответствующая собственная функция оператора $V(z)$. 
Достаточность. Пусть $\lambda=-1$ является собственным значением оператора $V(z)$ и $\varphi$ - соответствующая собственная функция, т.е.

$$
V(z) \varphi(x)=-\varphi(x)
$$

Тогда

$$
f(x)=\frac{\varphi(x)}{u(x)-z} \in L_{2}\left(T^{\nu}\right)
$$

и $f \neq 0$, при этом функция $f(x)$ удовлетворяет уравнению (4.3).

Из предложения 4.1 и теоремы Фредгольма [7] следует

ПреДЛОЖЕНИЕ 4.2. Число $z \in \mathbb{C} \backslash\left[m_{\nu}, M_{\nu}\right]$ является собственнъм значением оператора $U_{0}+U$ тогда и только тогда, когда $\Delta(z)=0$, где $\Delta(z)$ - детерминант Фредгольма оператора $I+V(z)$. Всякое собственное значение $z \in \mathbb{C} \backslash\left[m_{\nu}, M_{\nu}\right]$ оператора $U_{0}+U$ имеет конечную кратность.

Легко заметить, что $\sigma_{\mathrm{ess}}(\mathbf{h})=[m, M]$, где $m=\min u(x)=\alpha-6 \beta$ и $M=\max u(x)=$ $\alpha+6 \beta$.

ЛЕмма 4.3. Число $z \in \mathbb{C} \backslash[m, M]$ является собственным значением оператора $\mathbf{h}$ тогда и только тогда, когда

$$
\Delta_{0}(z)=\left(\frac{\beta_{0}}{\beta}\right)^{2}+\left\{\left(\left(\frac{\beta_{0}}{\beta}\right)^{2}-1\right)(z-\alpha)+2 \varepsilon_{1}\right\} \Im_{0}(z)=0,
$$

где $\Im_{0}(z)=\int_{T^{3}}(u(x)-z)^{-1} d x$.

ДоказАтеЛЬство. Оператор $\mathbf{h}$ является частным случаем оператора $U_{0}+U$. Для детерминанта Фредгольма $\Delta(z)$ оператора $I+V(z)$ имеем

$$
\Delta(z)=1+\sum_{n=1}^{\infty} \frac{d_{n}(z)}{n !}
$$

где

$$
d_{n}(z)=\int_{T^{3}} \ldots \int_{T^{3}}\left|\begin{array}{ccc}
k\left(\xi_{1}, \xi_{1}\right) & \ldots & k\left(\xi_{1}, \xi_{n}\right) \\
\vdots & \vdots & \vdots \\
k\left(\xi_{n}, \xi_{1}\right) & \ldots & k\left(\xi_{n}, \xi_{n}\right)
\end{array}\right| \frac{d \xi_{1} \ldots d \xi_{n}}{\prod_{i=1}^{n}\left(u\left(\xi_{i}\right)-z\right)} .
$$

Пользуясь явным видом ядра $k(x, s)=K(x, s)=\varepsilon_{1}+\varepsilon_{2}\left(h_{0}(x)+h_{0}(s)\right)$, можно показать, что $d_{n}(z) \equiv 0$ при любом $n \geqslant 3$.

Таким образом, имеем

$$
\Delta(z)=1+d_{1}(z)+\frac{d_{2}(z)}{2} .
$$

Нетрудно проверить, что

$$
\begin{aligned}
& d_{1}(z)=2\left(\varepsilon_{1} \Im_{0}(z)+\varepsilon_{2} \Im_{1}(z)\right), \\
& d_{2}(z)=2 \varepsilon_{2}^{2}\left(\Im_{1}^{2}(z)-\Im_{0}(z) \Im_{2}(z)\right) .
\end{aligned}
$$


Здесь

$$
\Im_{k}(z)=\int_{T^{3}} \frac{h_{0}^{k}(x) d x}{u(x)-z}, \quad k=0,1,2 .
$$

При $\beta \neq 0$ получим следующие равенства:

$$
\Im_{1}(z)=\frac{1-(\alpha-z) \Im_{0}(z)}{\beta}, \quad \Im_{2}(z)=\frac{\left((\alpha-z) \Im_{0}(z)-1\right)(\alpha-z)}{\beta^{2}} .
$$

Подставляя эти выражения в (4.5), (4.6) и упростив (4.4), получим, что $\Delta(z)=$ $\Delta_{0}(z)$. Отсюда и из предложения 4.2 следует доказательство леммы 4.3.

Всякая критическая точка $x^{\text {cr }}$ функции $u(x)$ имеет вид $x^{\mathrm{cr}}=(\pi m, \pi n, \pi k)$, где $m, n, k \in\{0,1,2\}$. Следовательно, значение гессиана $D_{u}(x)=-8 \beta^{3} \cos x_{1} \cos x_{2} \cos x_{3}$ функции $u(x)$ в любой критической точке $x^{\text {cr }}$ отлично от нуля, т.е. всякая критическая точка функции $u(x)$ является невырожденной. Отсюда следует, что при $z \rightarrow m-0$ и $z \rightarrow M+0$ интеграл $\Im_{0}(z)$ стремится к конечному пределу [9]. Положим

$$
A=\lim _{z \rightarrow m-0} \Im_{0}(z) \neq 0, \quad B=\lim _{z \rightarrow M+0} \Im_{0}(z) \neq 0 .
$$

Введем следующие обозначения:

$$
\begin{aligned}
& k_{1}=2\left\{\varepsilon_{1}-3\left(\left(\frac{\beta_{0}^{2}}{\beta}\right)^{2}-1\right) \beta\right\}+\left(\frac{\beta_{0}^{2}}{\beta}\right)^{2} A^{-1}, \\
& k_{2}=2\left\{\varepsilon_{1}+3\left(\left(\frac{\beta_{0}^{2}}{\beta}\right)^{2}-1\right) \beta\right\}+\left(\frac{\beta_{0}^{2}}{\beta}\right)^{2} B^{-1} .
\end{aligned}
$$

При $z \in \mathbb{C} \backslash[m, M]$ определим функцию $Q(z)=\Delta_{0}(z) / \Im_{0}(z)$. Очевидно, что $\Im_{0}(z) \neq 0$ при $z \notin[m, M]$. Отсюда следует, что если точка $z_{0} \notin[m, M]$ является нулем функции $\Delta_{0}(z)$, то она также является нулем функции $Q(z)$, и наоборот.

Лемма 4.4. Пусть $\varepsilon_{2} \neq 0, \beta_{0} \neq 0$. Тогда функция $Q(z)$ на полуоси $(-\infty, m)$ строго монотонно убывает от $+\infty$ до $k_{1}$, а на полуоси $(M, \infty)$ строго монотонно убъвает от $k_{2}$ до $-\infty$.

ДокАЗАТЕЛЬСтво. Положим $\omega=\left(\beta_{0} / \beta\right)^{2}$. Тогда

$$
Q(z)=\frac{\omega}{\Im_{0}(z)}+(\omega-1)(z-\alpha)+2 \varepsilon_{1} .
$$

Дифференцируя функцию $Q(z)$ по $z \in \Pi=\mathbb{R} \backslash[m, M]$, получим

$$
Q^{\prime}(z)=-\frac{\omega}{\Im_{0}^{2}(z)} \Im_{0}^{\prime}(z)+\omega-1 .
$$

В силу неравенства Шварца для $z \in \Pi$ имеем

$$
\left|\Im_{0}(z)\right| \leqslant \sqrt{\int_{T^{3}} \frac{d x}{(u(x)-z)^{2}}} \sqrt{\int_{T^{3}} 1^{2} d x}=\sqrt{\Im_{0}^{\prime}(z)} .
$$

Следовательно, $\Im_{0}^{2}(z) \leqslant \Im_{0}^{\prime}(z)$. Отсюда и из (4.8) вытекает, что $Q^{\prime}(z) \leqslant-1$, т.е. $Q^{\prime}(z)<0$ при $z \in \Pi$. Это означает, что функция $Q(z)$ на П является строго монотонно убывающей. 
Пусть теперь $z<m$. Легко заметить, что $0<\Im_{0}(z)<(m-z)^{-1}$ при $z \in(-\infty, m)$. Отсюда и из (4.7) имеем

$$
Q(z) \geqslant \omega(m-z)+(\omega-1)(z-\alpha)+2 \varepsilon_{1}=-z+2 \varepsilon_{1}+\omega m-(\omega-1) \alpha .
$$

Таким образом, $\lim _{z \rightarrow-\infty} Q(z)=+\infty$. Аналогично получаем, что $\lim _{z \rightarrow \infty} Q(z)=$ $-\infty$. Учитывая в равенстве (4.7) конечность значений пределов $A=\lim _{z \rightarrow m-0} \Im_{0}(z)$ и $B=\lim _{z \rightarrow M+0} \Im_{0}(z)$, имеем $k_{1}=\lim _{z \rightarrow m-0} Q(z)$ и $k_{2}=\lim _{z \rightarrow M+0} Q(z)$.

Это означает, что функция $Q(z)$ на полуоси $(-\infty, m)$ (на полуоси $(M, \infty))$ строго монотонно убывает от $+\infty\left(\right.$ от $\left.k_{2}\right)$ до $k_{1}($ до $-\infty)$.

Tеорема 4.1. Пусть $\varepsilon_{2} \neq 0, \beta_{0} \neq 0$. Тогда:

а) если $k_{1}<0$ и $k_{2}>0$, то оператор $\mathbf{h}$ вне существенного спектра имеет два собственных значения $z_{1}$ и $z_{2}$, причем $z_{1}$ лежит ниже, а $z_{2}$ - выше существенного спектра;

б) если $k_{1}<0 u k_{2} \leqslant 0\left(k_{1} \geqslant 0 u k_{2}>0\right)$, то оператор $\mathbf{h}$ вне существенного спектра имеет единственное собственное значение $z=z_{0}$, причем оно лежит ниже (выше) существенного спектра;

в) если $k_{1} \geqslant 0$ u $k_{2} \leqslant 0$, то у оператора $\mathbf{h}$ вне существенного спектра нет собственных значений, m.е. $\sigma(\mathbf{h})=\sigma_{\mathrm{ess}}(\mathbf{h})=\sigma\left(\mathbf{h}_{0}\right)$.

ДоКАЗАТЕЛЬСтво непосредственно следует из лемм 4.3 и 4.4 .

Если на решетке $\mathbb{Z}^{3}$ отсутствует примесь, то оператор $H^{\mathrm{t}}$ совпадает с оператором триплета $H_{0}^{\mathrm{t}}$ на беспримесной решетке [2], действующим по формуле $H_{0}^{\mathrm{t}} f(x, y)=$ $(u(x)+u(y)) f(x, y), f \in L_{2}^{\mathrm{as}}$. При этом $\sigma\left(H_{0}^{\mathrm{t}}\right)=[2 m, 2 M]$. Для спектра гамильтониана $H^{\mathrm{t}}$ имеет место

Tеорема 4.2. Пусть $\varepsilon_{2} \neq 0, \beta_{0} \neq 0$. Тогда:

а) если $k_{1}<0$ и $k_{2}>0$, то существуют числа $z_{1}<m u z_{2}>M$ такие, что $\Delta_{0}\left(z_{1}\right)=0$ и $\Delta_{0}\left(z_{2}\right)=0$, для которых

$$
\sigma\left(H^{\mathrm{t}}\right)=\sigma\left(H_{0}^{\mathrm{t}}\right) \cup\left[m+z_{1}, M+z_{1}\right] \cup\left[m+z_{2}, M+z_{2}\right] \cup\left\{2 z_{1}, 2 z_{2}, z_{1}+z_{2}\right\},
$$

m.e.

$$
\sigma_{\mathrm{ess}}\left(H^{\mathrm{t}}\right)=\sigma\left(H_{0}^{\mathrm{t}}\right) \cup\left[m+z_{1}, M+z_{1}\right] \cup\left[m+z_{2}, M+z_{2}\right] ;
$$

б) если $k_{1}<0$ и $k_{2} \leqslant 0\left(k_{1} \geqslant 0 u k_{2}>0\right)$, то существует число $z_{0}<m\left(z_{0}>M\right)$ такое, что $\Delta\left(z_{0}\right)=0$, для которого

$$
\sigma\left(H^{\mathrm{t}}\right)=\sigma\left(H_{0}^{\mathrm{t}}\right) \cup\left[m+z_{0}, M+z_{0}\right] \cup\left\{2 z_{0}\right\},
$$

m.e.

$$
\sigma_{\text {ess }}\left(H^{\mathrm{t}}\right)=\sigma\left(H_{0}^{\mathrm{t}}\right) \cup\left[m+z_{0}, M+z_{0}\right], \quad \sigma_{\text {disc }}\left(H^{\mathrm{t}}\right)=\left\{2 z_{0}\right\} ;
$$

в) если $k_{1} \geqslant 0$ u $k_{2} \leqslant 0$, то спектр оператора $H^{\mathrm{t}}$ совпадает со спектром операmopa $H_{0}^{\mathrm{t}}$, m.e.

$$
\sigma\left(H^{\mathrm{t}}\right)=\sigma\left(H_{0}^{\mathrm{t}}\right)
$$

4 Теоретическая и математическая физика, т. 149, № 2, 2006 г. 
ДокАЗАТЕЛЬСтво. В гильбертовом пространстве $L_{2}\left(T^{3}\right) \otimes L_{2}\left(T^{3}\right)$ рассмотрим самосопряженный оператор $W=\mathbf{h} \otimes E+E \otimes \mathbf{h}$. Для спектра оператора $W$ имеем следующее равенство [5]:

$$
\sigma(W)=\sigma(\mathbf{h})+\sigma(\mathbf{h}) .
$$

С другой стороны, учитывая равенство

$$
\begin{aligned}
H^{\mathrm{t}} f(x, y)=( & \left.u(x) f(x, y)+\int_{T^{3}} K(x, s) f(s, y) d s\right)+ \\
& +\left(u(y) f(x, y)+\int_{T^{3}} K(y, t) f(x, t) d t\right),
\end{aligned}
$$

мы можем установить унитарную эквивалентность операторов $H^{\mathrm{t}}$ и $W$. Следовательно, пользуясь равенством (4.9), имеем

$$
\sigma\left(H^{\mathrm{t}}\right)=\sigma(W)=[2 m, 2 M] \cup\left(\bigcup_{\lambda \in \sigma_{\mathrm{disc}}(\mathbf{h})}[m+\lambda, M+\lambda]\right) \cup D_{0},
$$

где $D_{0}=\left\{\omega: \omega=\lambda+\mu \notin[2 m, 2 M], \lambda, \mu \in \sigma_{\text {disc }}(\mathbf{h})\right\}$.

Теперь рассмотрим операторы $\widehat{H}_{1}$ и $\widehat{H}_{2}$ такие, что

$$
\begin{array}{ll}
\widehat{H}_{1} f(x, y)=(u(x)+u(y)) f(x, y)+\int_{T^{3}} K(x, s) f(s, y) d s, & f \in L_{2}(\Omega \times \Omega), \\
\widehat{H}_{2} f(x, y)=(u(x)+u(y)) f(x, y)+\int_{T^{3}} K(y, t) f(x, t) d t, & f \in L_{2}(\Omega \times \Omega) .
\end{array}
$$

Тогда $\widehat{H}_{1}=\mathbf{h} \otimes E+E \otimes \mathbf{h}_{0}$ и $\widehat{H}_{2}=\mathbf{h}_{0} \otimes E+E \otimes \mathbf{h}$, где $\mathbf{h}_{0}$ - оператор умножения на функцию $u(x)$ в $L_{2}(\Omega)$, т.е. $\mathbf{h}_{0} \varphi(x)=u(x) \varphi(x), \varphi \in L_{2}(\Omega)$. Отсюда $\sigma_{0}=\sigma\left(H_{0}^{\mathrm{t}}\right)=$ $[2 m, 2 M]$ и

$$
\begin{aligned}
& \sigma_{1}=\sigma\left(\widehat{H}_{1}\right)=\sigma(\mathbf{h})+\sigma\left(\mathbf{h}_{0}\right)=[2 m, 2 M] \cup\left(\bigcup_{\lambda \in \sigma_{\mathrm{disc}}(\mathbf{h})}[m+\lambda, M+\lambda]\right), \\
& \sigma_{2}=\sigma\left(\widehat{H}_{2}\right)=\sigma\left(\mathbf{h}_{0}\right)+\sigma(\mathbf{h})=\sigma_{1} .
\end{aligned}
$$

Используя теорему 3.3 , находим, что

$$
\sigma_{\text {ess }}\left(H^{\mathrm{t}}\right)=\sigma\left(H_{0}^{\mathrm{t}}\right) \cup\left(\bigcup_{\lambda \in \sigma_{\text {disc }}(\mathbf{h})}[m+\lambda, M+\lambda]\right), \quad \sigma_{\text {disc }}\left(H^{\mathrm{t}}\right)=D_{0} .
$$

Применяем результаты теоремы 4.1 и этим завершаем доказательство теоремы.

Теорема 4.3. Если выполнено неравенство

$$
A D<\frac{\sqrt{5}-1}{2}
$$

где $D=\left|\varepsilon_{1}\right|+12\left|\varepsilon_{2}\right|>0$, mo

$$
\sigma(\mathbf{h})=\sigma\left(h_{0}\right), \quad \sigma\left(H^{\mathrm{t}}\right)=\sigma\left(H_{0}^{\mathrm{t}}\right) .
$$


Доказательство. Обозначим через $D$ наибольшее значение функции $|K(x, y)|$ при $x, y \in T^{3}$. Нетрудно проверить, что $D=\left|\varepsilon_{1}\right|+12\left|\varepsilon_{2}\right|$. Следовательно, для членов $d_{1}(z)$ и $d_{2}(z)$ детерминанта Фредгольма имеем оценки $\left|d_{1}(z)\right| \leqslant D\left|\Im_{0}(z)\right|$ и $\left|d_{2}(z)\right| \leqslant 2 D^{2} \Im_{0}^{2}(z)$. Учитывая конечность пределов $A=\lim _{z \rightarrow m-0} \Im_{0}(z)$ и $B=$ $\lim _{z \rightarrow M+0} \Im_{0}(z)$, мы можем определить значение функции $\Im_{0}(z)$ в точках $z=m$ и $z=M$ по непрерывности:

$$
\Im_{0}(m)=\lim _{z \rightarrow m-0} \Im_{0}(z)=A>0, \quad \Im_{0}(M)=\lim _{z \rightarrow M+0} \Im_{0}(z)=B<0 .
$$

Таким образом, функция $\Im_{0}(z)$ становится непрерывной на полуинтервалах $(-\infty, m]$ и $[M, \infty)$. Из очевидного равенства $\Im_{0}(m)=-\Im_{0}(M)$, т.е. $A=-B$, получим, что

$$
\left|d_{1}(z)\right| \leqslant A D, \quad\left|d_{2}(z)\right| \leqslant 2(A D)^{2} .
$$

Пусть выполнено условие (4.11). Тогда

$$
A D+(A D)^{2}<1
$$

При этом условии функция $\Delta_{0}(z)$ на $\Pi=\mathbb{R} \backslash[m, M]$ не обращается в нуль, так как из $\Delta_{0}(z)=0$ следует, что $d_{1}(z)+d_{2}(z) / 2=-1$. С другой стороны, с учетом неравенств (4.12) и (4.13) мы имеем $\left|d_{1}(z)+d_{2}(z) / 2\right|<1$. Это означает, что функция $\Delta_{0}(z)$ на П не может обращаться в нуль. Тогда в силу леммы 4.3 оператор $\mathbf{h}$ вне существенного спектра не имеет ни одного собственного значения, т.е. $\sigma(\mathbf{h})=\sigma\left(\mathbf{h}_{0}\right)$. Отсюда и из (4.10) получим, что $\sigma\left(H^{\mathrm{t}}\right)=\sigma\left(H_{0}\right)$.

\section{Список литературы}

[1] D. Mattis, Rev. Mod. Phys., 58 (1986), 361-379; E. Lieb, Phys. Rev. Lett., 62 (1989), 1201-1204; A. M. Tsvelick, P. B. Wiegman, Adv. Phys., 32 (1983), 453-713.

[2] Б. В. Карпенко, В. В. Дякин, Г. А. Будрина, $Ф M M, 61: 4$ (1986), 702-706.

[3] Ю. А. Изюмов, Ю. Н. Скрябин, Статистическая механика магнитно-упорядоченных систем, Наука, М., 1974.

[4] Л. Д. Ландау, Е. М. Лифшиц, Квантовая механика. Нерелятивистская теория, Наука, М., 1974.

[5] М. Рид, Б. Саймон, Методы современной математической физики, m. 1. Функиионалъный анализ, Мир, М., 1977.

[6] Ю.Х. Эшкабилов, Докл. АН РУз, 2006.

[7] Ф. Трикоми, Интегралъные уравнения, ИЛ, М., 1960.

[8] М. Рид, Б. Саймон, Методы современной математической физики, m. 4. Анализ операторов, Мир, М., 1982.

[9] М. Ю. Федорюк, Асимптотика: интеграль и рядъ, Наука, М., 1987.

Поступила в редакцию 2.12.2003, после доработки 10.04.2006 